\title{
Discovery of Five New X-ray-Selected BL Lacertae Objects and Three New Quasars ${ }^{1}$
}

G.Z. Xie, ${ }^{2}$ W. Brinkmann ${ }^{3}$ G.W. Cha, ${ }^{2}$ S. Laurent-Muehleisen, ${ }^{4}$ Y. H. Zhang, ${ }^{2}$ K. H. Li, ${ }^{2}$ J.M. Bai, ${ }^{2}$ and F.K. Liu ${ }^{2}$

${ }^{2}$ Yunnan Observatory, The Chinese Academy of Sciences, Kunming 650011, China

${ }^{3}$ Max-Planck-Institute für Extraterrestrische Physik, D-8046 Garching, Germany

${ }^{4}$ Department of Astronomy and Astrophysics, The Pennsylvania State University, University Park, PA 16802, USA

Through combined ROSAT and VLA observations, we have identified $19 \mathrm{BL}$ Lac/quasar candidates. In 1994 December, 1995 January and October, and 1996 January 1996, we obtained spectra of all 19 candidates using the 2.16-m telescope of Beijing Astronomical Observatory. The dispersion used is $195 \AA \mathrm{mm}^{-1}$, which yields a dispersion of about $4.65 \AA$ pixel $^{-1}$, and the wavelength coverage is 3500-7800 $\AA$. Five of these objects are uniformly featureless, and we identify them as BL Lac objects. Three new quasars are also identified. Table 1 presents VLA positions and redshifts for the 5 new BL Lac objects and three new quasars. By checking them in the NASA/IPAC Extragalactic Database (NED) and Véron-Cetty \& Véron's (1993) Catalogue of Quasars and AGNs, and other recent reports about discovery of new BL Lac objects and quasars, we find that these sources are previously unreported.

Table 1. Positions and Redshifts of New BL Lac Objects and Quasars

\begin{tabular}{ccccl}
\hline \hline Name & $\alpha_{2000}$ & $\delta_{2000}$ & $z$ & Identification \\
\hline $0030+380$ & 003018.9 & 380401.7 & $\ldots$ & BL Lac \\
$0110+415$ & 011004.8 & 414950.7 & $\ldots$ & BL Lac \\
$0123+342$ & 012308.7 & 342050.5 & $\cdots$ & BL Lac \\
$0136+390$ & 013632.7 & 390600.0 & $\ldots$ & BL Lac \\
$0159+104$ & 015934.4 & 104706.4 & $\ldots$ & BL Lac \\
$0027+451$ & 002742.3 & 451457.1 & $0.420 \pm 0.004$ & QSO \\
$0314+244$ & 031402.7 & 244430.9 & $0.427 \pm 0.004$ & QSO \\
$2313+101$ & 231355.7 & 101909.8 & $0.447 \pm 0.005$ & QSO \\
\hline
\end{tabular}

${ }^{1}$ This work is supported by the National Natural Science Foundation of China. 


\section{References}

Véron-Cetty, M.-P., \& Véron, P. 1993, ESO Scientific Report, No. 13. 